\title{
Experimental Determination of Magnetohydrodynamic Sealwater Thruster Performance in a Two Tesla Test Facility
}

\author{
ANU/CF-76576 \\ by \\ DE92. 019042 \\ A?
}

Basil Picologlou, Ezzat Doss, and Dave Black

Engineering Physics Division

Argonne National Laboratory

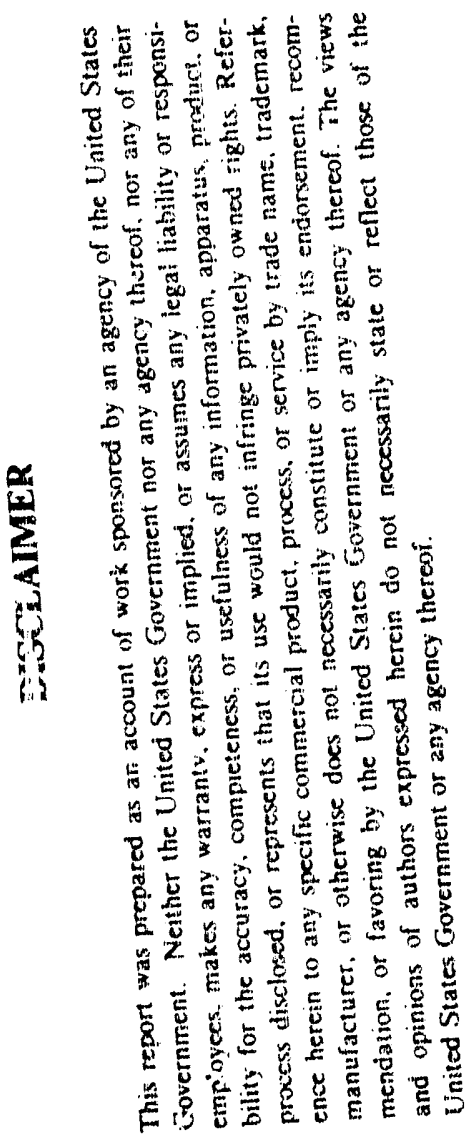

and

W. Christopher Sikes

Advanced Propulsion Technology

Newport News Shipbuilding and Dry Dock Company

$27^{\text {th }}$ Intersociety Energy Conversion

Engineering Conference (IECEC)

San Diego, California

August 3-7, 1992

The submilted maniscript has been partially authored try a contractor nt the U.S. Gowernment

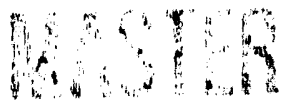
under Contract number W-31-1199.Eng-38. Accordingly, the U.S. Govemment retans a nonexclusive, royalty'-free license to publish or reproduce the published form of this contribution. or allow others to dD so, for U.S. Government purposes. 


\title{
EXPERIMENTAL DETERMINATION OF MAGNETOHYDRODYNAMIC SEAWATER THRUSTER PERFORMANCE IN A TWO TESLA TEST FACILITY
}

\author{
Basil Picologlou, Ezzat Doss, and Dave Black \\ Engineering Physics Division \\ Argonne National Laboratory \\ and \\ W. Christopher Sikes \\ Advanced Propulsion Technology \\ Newport News Shipbuilding and Dry Dock Company
}

\begin{abstract}
A two Tesla test facility was designed, built, and operated to investigate the performance of magnetohydrodynamic (MHD) seawater thrusters. The results of this investigation are used to validate MHD thruster performance computer models.

The facility test loop, its components, and their design are presented in detail. Additionally, the test matrix and its rationale are discussed. Finally, representative experimental results of the test program are presented, and are compared to pretest computer model predictions.

Good agreement between predicted and measured data has served to validate the thruster performance computer models.
\end{abstract}

\section{INTRODUCTION}

The idea of using MHD seawater propulsion for surface ships and submarines has beer examined in the past by many investigators. $[1.5]$ However, it has been demonstrated that the attractiveness of this propulsion concept is dependent on the development of high magnetic nux density superconducsing magneis. [이

Recent advances in superconductors have renewed interest in using this propulsion concept. The potential benefits of this forward-thinking propulsion system include reduced nosse (from the elimination of rotating machinery required to support conventional propulsion) and potential for operating at higher speeds because an MHD thruster, unlike a propeller, is not cavitation limited. Moreover, the arrangement of components needed for MHD propulsion does not require a fixed stack-up, thus allowing greater architectural flexibility for the shipbuilder. To explore these potential benefits, conceptual designs of surface ships and submarines are being pursued. ${ }^{(7)}$ Crucial to this process are validated design tools that address the performance characteristics of MHD thrusters as a function of their geometry and operating parameters.

A collaborative effort between Newport News Shipbuilding (NNS) and Argonne National Laboratory (ANL) was established to demonstrate the feasibility of MHD seawater propulsion through development of computer models of thruster performance and experiments to validate adequacy of the developed models. The first phase of this multiyear/multiphase program, the development of a design oriented, lumped parameter. systems code that predicts the performance of an MHD thruster, was completed in 1990.

The focus of the scoond phase is experimental validation of the thruster performance code and 
identification through testing of any phenomena which may limit the theoretically predicted thruster performance thereby impacting the attractiveness of this propulsion system for shipboard applications. To accomplish this in a cost-effective manner, a test facility has been built at ANL using an existing 2 Tesla conventional electromagnet.

\section{TEST FACILITY LAYOUT}

The test facility, Figure 1, consists of a 2 Tesla conventional electromagnet. a test section, gas separator, a loop pump, polyvinylchloride (PVC) piping and related fittings. Additional systems support both loop operation and data acquisition.

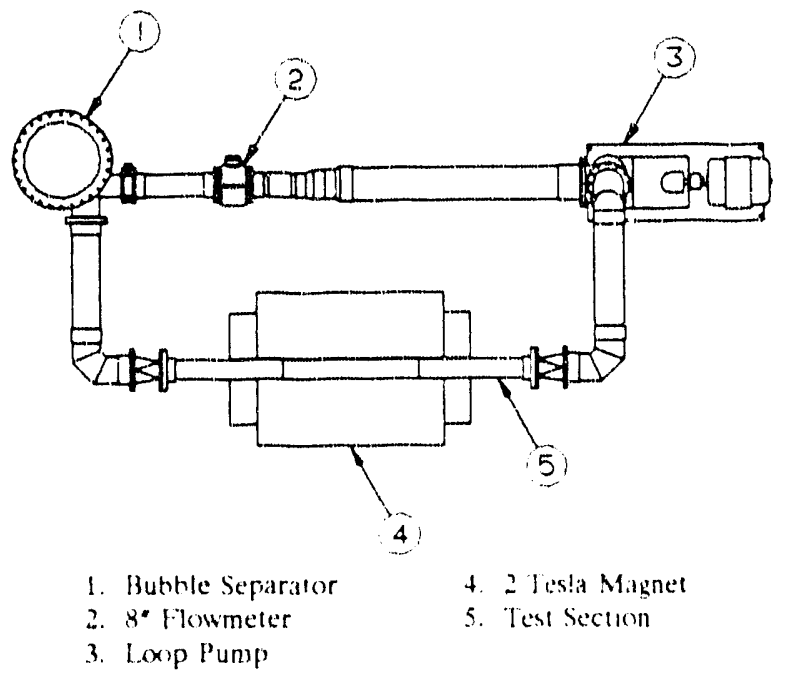

Figure 1: Test Facility Lityout

The purpose of the test loop is to circulate the working fluid through the test section, located in the two Tesla magnetic field. The test loop also provides for the removal of the electrolytically generated hydrogen bubbies from the working fluid.

WORKING FLUID - Aqueous solution of sodium chloride $(\mathrm{NaCl})$ was selected as the working medium in lieu of Instant Ocean ${ }^{\text {TM }}$ because the $\mathrm{NaCl}$ solution does not contain additional dissolved salts whose precipitates (e.g. calcium hydroxide $-\mathrm{Ca}[\mathrm{OH}]_{2}$ and magnesium hydroxide $\left.\mathrm{Mg}(\mathrm{OH})_{2}\right)$ might foul the loop during operation. Increased $\mathrm{NaCl}$ concentrations beyond values characteristic of seawater are used to enhance electrical conductivity and thus achieve test operating conditions closer to prototypic than would have been possible with conductivities representative of seawater alone.

All loop components were selected to he compatible with both the working fluid and the products of electrolysis such as: hydrogen gas $\left(\mathrm{H}_{2}\right)$, sodium hypochlorite $(\mathrm{NaClO})$, and hypochlorous acid $(\mathrm{HClO}) \cdot|8|$

MAGNET - An existing 2 Tesla, iron core, conventional electromagnet with a uniform magnetic field volume of $8^{\prime \prime} \times 30^{*} \times 50^{*}$ is used in the test setup. This 67 ton water-cooled electromagnet was selected because the magnetic field can he oriented either vertically or horizontally to observe the effect of gravity on bubble evolution and MHD thruster performance.

TEST SECTYON - Sheets of optically-clear polycarbonate resin were solvent welded with methylene chloride $\left(\mathrm{CH}_{2} \mathrm{Cl}_{2}\right)$, strengthened with brass screws, and sealed with a silicone rubber adhesive to form the $6^{\prime \prime} \times 6^{\prime \prime}$ $x 12^{\prime}$ test section. This material was selected to enable visual observation/measurement of the electrolyticallygenerated hydrogen bubbles at the cathode. Seven pressure taps were installed along the $50^{n}$ active length of the test section (that portion of the test section where the electrodes are located) to measure the internal pressure distribution. Each electrode was segmented into five parts enabling specific measurement of both applied current and voltage to beller ascertain the impact of fringing current on thruster performance. The anode segments are a mixedoxide coating (ruthenium) on a $1 / 4^{\prime \prime}$ thick titanium substrate and the cathode segments are titanium plates of the same thickness. A removable lid was installed to allow for electrode maintenance. The non-active portion of the test section was included in the design, along with an inlet flow straightener. 10 provide a well developed velocity protile for the active section of the thruster.

BUBBLE SEPARATOR - A bubbie separator was included in the loop to assist in the separation of hydrogen hubbles from the working fluid. The eparator tank design is based on a cyclone principle commonly used to separate solids and liquids from gas streams. In this application, the centrifugal tield moves the hydrogen bubbles to the center where they rise, enter the standpipe gas space, are diluted by nitrogen gas below the nammahility limul, and are exhausted to the atmosphere. The tank is 30" in diameter and $7+4: 2$ tall. Inlet and outet connections were installed off-axis to generate the centrifugal field required for separation. A flanged lid was provided to facilitate coating the incerior of the lank with a synthetic rubber corrosion preventative. Elliptical heads were installed to facilitate gas removal and loop drainage.

A 33" tall, 4" diameter, 38" thick acrylic pipe is installed, via adapter, at the top of the separator to enable loop operators to monitor the working fluid level in the loop, facilitate the injection of nitrogen into the system, and to provide an expansion volume. Loop pressurization. hydrogen dilution, and venting is accomplished through appropriate standpipe penetrations.

LOOP PUMP. The loop pump is a $30 \mathrm{kw}(40 \mathrm{hp})$ horizontal end-suction $12 " \times 10^{m}$ centrifugal pump whose motor is driven hy a variable frequency speed controller. This controller enables testing at fluid velocities in the range of $2.8 \mathrm{~m} / \mathrm{s}$ independently of current appleed ti) the electrodes.

PIPING - Schedule 40 PVC piping was used in the lest loop. Pipe sizes were established by the pump discharge $\left(10^{n}\right)$, the flowmeter $\left(8^{n}\right)$, and the pump suction (12"). Fiberglass reinforced piping transitions (10" round to $6^{\prime \prime}$ square) were installed to reduce flow losses inro/out of the test section. An $8^{*}$ flexible connection was installed between the two fixed points in the system, the loop pump and the separator tank, to accommodate changes in pipe iengin cue to variations in iemperaiure, io sciuce vibatioñ in the loop from the pump, and to accommodate any 
misalignment of loop components.

\section{INSTRUMENTATION}

Two types of instrumentation were used in the test program. The first type includes the instrumentation that supplies the data necessary to satisfy the primary objective of the test program, i.e. the determination of thruster performance. The second type includes all instrumentation necessary to monitor loop performance and carry out the various operations during preparation for loop start-up, testing, and post-test loop shutdown. The instruments in the former group include:

- An $8^{n}$ electromagnetic flowmeter 10 measure flowrate (and thus, flow velocity) in the thruster.

- A working fluid conductivity analyzer.

- Two differential pressure iransducers/transmitters along with an appropriate valving system, to measure the pressure distribution in the test section.

- Ten current shunts for the measurement of the individual electrical currents supplied to the five anode segments and the corresponding currents collected from the five cathode segments.

- A multichannel integrating digital voltmeter to sample the potential difference across the five anode/cathode pairs to ensure that they are driven by the same differential voltage.

- A thermocouple to measure fluid temperature.

All signals from these instruments, as well as others that provide pressure distribution along the loop are sampled, on demand, by a minicomputer-driven data acquisition system (DAS) and recorded in memory. In addition to measurement of electrical and mechanical thruster parameters, the fully transparent test article provided a unique opportunity for visual observation of the bubbles and optical measurement of their characteristics via high speed video recording. Visual observation was done over a length of $47^{\prime \prime}$ downstream of the electrodes whereas optical measurements were carricd out $33^{\prime \prime}$ downstream of the clectrode edge.

\section{PRE-TEST ANALYSIS}

A pre-test analysis was performed to support the design of the loop and provide guidance in establishing a test matrix, using the system code developed in Phase I. The MHD propulsion system code includes computer models for different components of the propulsion system. Among those components are models of the MHD thruster, nozzle, diffuser, pipe, and drag for submarines and surface ships.

The MHD inruster model couples a onc. dimensional hydrodynamic flow model to a two-dimensional electrical model. The equations used in the hydrodynamic model are the usual one-dimensional equations for conservation of mass:

$$
m=\rho A u
$$

and conservation of momentum.

$$
\rho u \frac{d u}{d x}=-\frac{d p}{d x}+J \times B-\frac{f \rho u^{2}}{2 d}
$$

The two-dimensional electrical model solves Maxwell's equation for the electrical field potential in conjunction with Ohm's law which for an MHD thruster can be written:

$$
J=\sigma(E-U B)
$$

The resulting modified Laplace equation for the electrical potential given by:

$$
\frac{\partial^{2} \phi}{\partial x^{2}}+\frac{\partial^{2} \phi}{\partial y^{2}}=0
$$

is solved using an overrelaxation procedure. This twodimensional electrical model is used to predict the current in the fringing region of the magnct near the ends of the electrodes and to determine the electrical end losses in that region. Details of the MHD thruster model are given in reference [6]. The MHD system code, including the thruster model, has been used to design loop components, define optimum operating conditions, assist in the selection of instrumentation for the individual components, and predict the overail characteristics of the test loop.

A parametric sweep was performed using the following variables:

- Separator toss coefficien, $\wedge=0-1.25$

- Working fluid conductivity $\sigma=4.5-22.5 \mathrm{~S} / \mathrm{m}$

- Test section velocity $u=2-8 \mathrm{~m} / \mathrm{s}$

- Load factor $K=1.1-30$

Dutput values include voltage, current, working fluid, loop component velocity and pressure drop, and pressure loss/recovery in the test section. The actual magnetic flux distribution of the 2 Tesla magnet was used in the facility model.

\section{TEST MATRIX}

The test program consisted of the following measurements:

1. Measurement of pressure distribution in the test section with zero magnetic field over a velocity range of $2-8$ $\mathrm{m} / \mathrm{s}$ to determine the haseline frictional losses.

2. Measurement of pressure distribution in the test section at 2 Tesla wish no current supplied to the thruster 
over a velocity range of $2.8 \mathrm{~m} / \mathrm{s}$ to determine the modification of the pressure distribution by the magnetic field alone.

3. Measurement of the volt-ampere characteristics of the thruster at zero magnetic field to determine the total electrochemical potential, confirm the conductivity of the working fluid, and obtain preliminary evidence on the effect of bubbles on the electrical performance of the thruster. Data were obtained at two different fluid velocities and current densities in the range of $67.780 \mathrm{~A} / \mathrm{m}^{2}$. This current density range spans the expected current density range of a full-size marine MHD thruster.

4. Measurements of the thruster performance at a magnetic field of 2 Tesla over a velocity range of $2.8 \mathrm{~m} / \mathrm{s}$ and a current density range of $70.820 \mathrm{~A} / \mathrm{m}^{2}$.

Measurements 1 through 4 were obtained at (wo) fluid conductivities, 4.5 and $22.5 \mathrm{~S} / \mathrm{m}$, with a horizontal magnetic field, and one conductivity, $22.5 \mathrm{~S} / \mathrm{m}$, with a vertical magnetic field. The test matrices included more than 400 data scans tor different operating conditions and pressure differentials.

\section{RESULTS AND DISCUSSION}

The measured Volt-Ampere (V-I) characteristics of the enter pair of thruster electrodes $1.30^{n}$ in length) are shown in Figure 2 for differeme conductivities, now velocities, and magnetic ficld orientations. The straight line character of the V.I characteristics indicates that, for thruster-relevant current densities, the generation of hydrogen bubbles at the cathode has a negligible effect on the current transport across the working fluid and that the single selectrode potentials (SEP's) are independent of the current density. The fact that the V-I characteristics are independent of both the velocity and the magnetic field orientation provides further support to the first conclusion. The fuid conductivity calculated from the slope of the V-I characteristics is in excellent agreement with the value measured by the conductivity probe. Finally, the sum of the SEP's at the anode and cathode, given by the $y$ intercept of the V-I characteristic $(2.6 \mathrm{~V}$ for $4.5 \mathrm{~S} / \mathrm{m}$ and $2.5 \mathrm{~V}$ for $22.5 \mathrm{Sim}$ ), is in excellent agreement with the sum of the single electrode potentials provided by Eltech, the electrode manufacturer. Eliech quoted a measured SEP of 1.1 volts for the anode and an estimated value, based on literature. of $1.5 \mathrm{~V}$ for the cathode. These figures are experted to increase slightly with increased current density and decreasing salt concentration. In their totality, the V-I characteristics results demonstrate that for the current densities of interest to MHD seawater propulsion, well established constant values of SEP's and fluid conductivity are the only electrical material properties that enter in the calculation of current transport and thruster performance.

Since the electrodes are segmented, the measurement of individual currents permits the determination of fringing current effects at the electrode ends. Figure 3 shows the measured and calculated average current densities over the five electrode segments. The figure indicates that the fringing current effects are confined w rela!ively smal! aroas and: as such, they are.

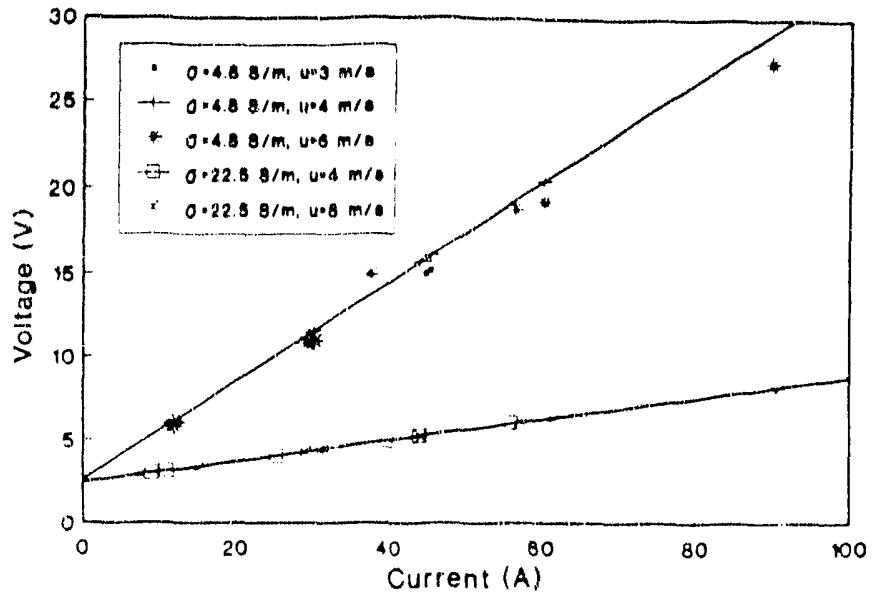

Figure 2: V.I Characteristics

expected to have a small effect on the performance of thrusters with moderately large length to width ratios in load factors greater that $1.5 .^{[4]}$

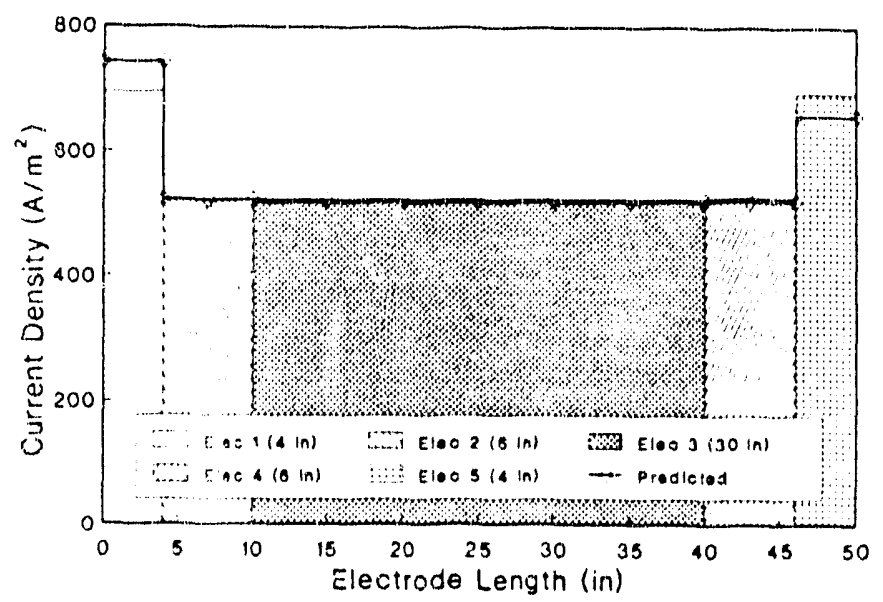

Figure 3: Current Distribution Among the Electrode Segments

The measured pressure difference over the 30 " center electrode was used to determine the degree to which the performance of the thruster follows theoretical predictions. Specifically, the measured pressure difference during thrust producing operation is reduced by the measured pressure difference at the same velocity and magnetic field, but at zero current. The resultant MHD pressure rise is normalized by the eneoretical pressure rise calculated on the hasis of the measured current, and plotied as a funcrion of the current densly as indicaled in Figure 4. As expected, there is good agreement hetheen measured and calculated pressure rise (thrust).

The thruster efficiency, defined as the ratio of the mechanical power generated hy the thruster fo the electrical power suppied the thruster is gisen hy:

$$
\eta=\frac{\Delta p Q}{\Delta V I}
$$

where $\Delta p$ is the pressure rise along the thruster, $Q$ is the volumetric flow rate. $\Delta V$ is the potential difference across the electrodes. and $I$ is the electric currint. 


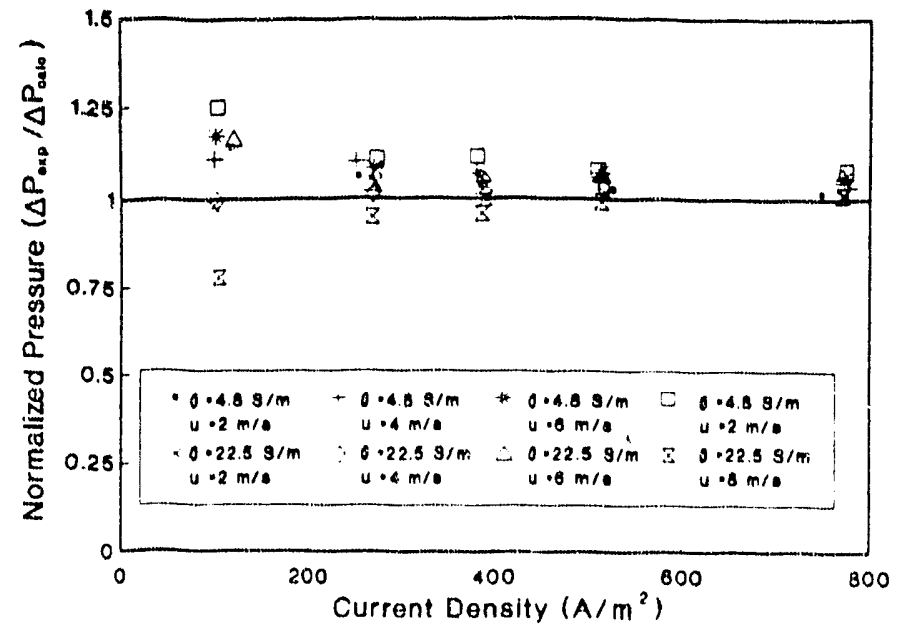

Figure 4: Comparison of Calculated and Measured Pressure Rise vs. Current Density

In Figure 5 , the measured total efficiency for $\sigma=22.5 \mathrm{~S} / \mathrm{m}$ and $u=2 \mathrm{~m} / \mathrm{s}$ is plotted against the load factor $\mathrm{K}$.

$$
K=\frac{\Delta V / d}{u B}
$$

where, $d$ is the electrode separation, $B$ is the magnetic thux density, and $u$ is the fluid velocity. As shown in Figure 5 , a total efficiency of about $9 \%$ was achieved under these conditions. Figure 5 also shows that the total efficiency is given by the ideal thruster efficiency (equal to $1 / \mathrm{K}$ ) reduced by frictional drag and SEP losses. Since both the losses and the ideal thruster efficiency are easily calculated, the performance of MHD thruster can be predicted with confidence. The calculation of the effect of end losses on efficiency is more complicated, but such losses are of little practical importance unless thrusters with magnetic flux densities in excess of 10 Tesla and for thrusters operating near load factors $K=1$ are under consideration. ${ }^{[9]}$

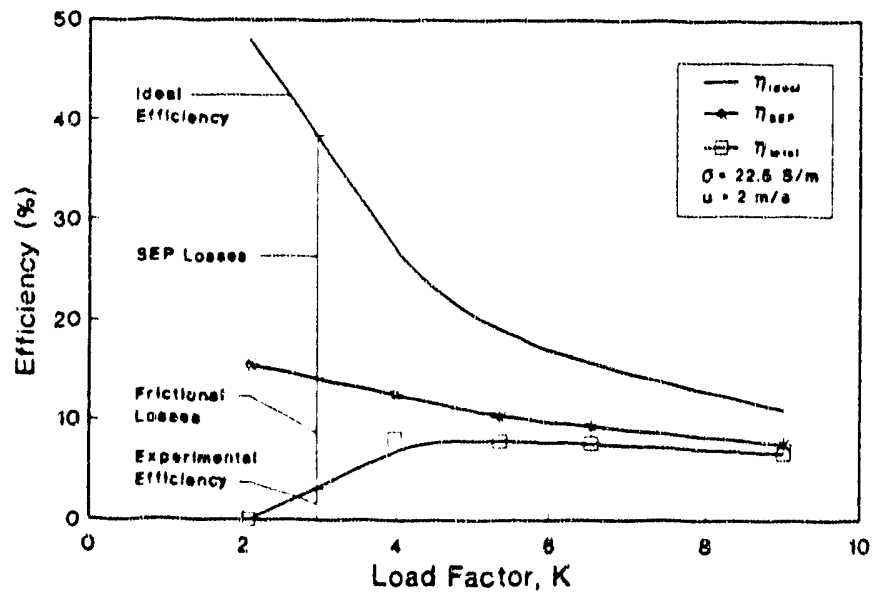

Figure 5: Thruster Efficiency and Losses vs. Load Factor

The measured ideal thruster efficiency for different operating conditions, calculated from the measured total efficiencies and losses, is plotted against the load factor in Figure 6. As shown in this figure, there is good agreement between the experimentally determined ideal efficiencies and theoretical dependence of $1 / \mathrm{K}$.

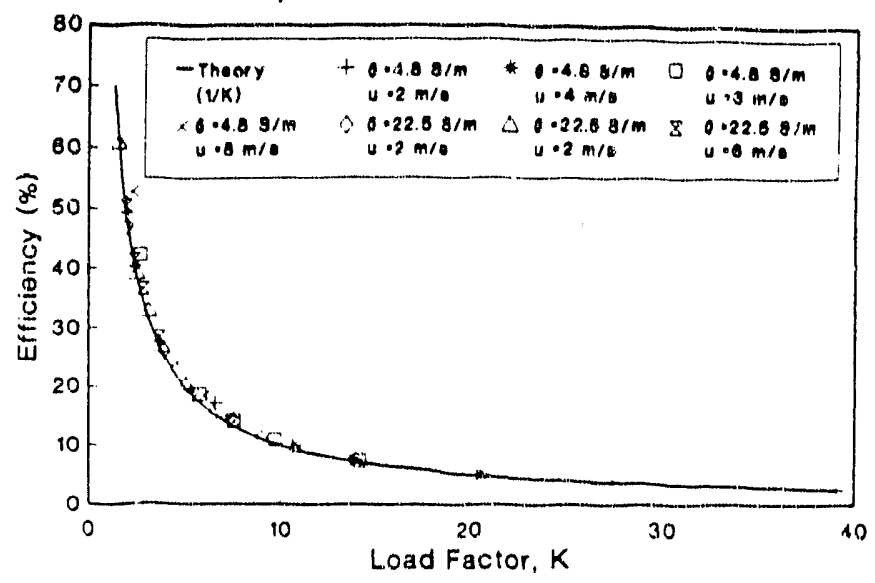

Figure 6: Measured (Ideal) Efficiency vs. Load Factor

As seen fro.n the above figures, the theoretical predictions are in good agreement with 1, measured values. Deviations can be attributed to experimental errors, given the fact that differential pressure measurements are as small as a few tenths of a psi for some of the cases. The results presented here are confined to the central portion of the thruster, away from the fringing iurrent areas. Measurements in these end regions are subject to large experimental error because the pressure differentials involved are small. Calculations have indicated that losses associated with such end effects are much smaller than frictional losses for the expected range of parameters of actual seawater thrusters.

As bited earlier, during the course of the test program, hoth visual observations and optical measurements of the hydrogen hubbles generated at the cathode were made. A detailed discussion of these measurements is beyond the scope of this paper. It is only mentioned that the presence of hydrogen" bubbles downstream of the inruster is a function of both fluid velocity and current density. For example, at $500 \mathrm{~A} / \mathrm{m}^{2}$, bubbles appear only at fluid velocities below $7 \mathrm{~m}$ s, whereas at $250 \mathrm{~A} / \mathrm{m}^{2}$, bubbles appear only at fluid velocities below $3.5 \mathrm{~m} / \mathrm{s}$. Apparently, provided that the hydrogen production rate at the cathode is sufficiently low and the mass transfer rate away from the cathode is sufficiently high, hydrogen goes into solution immediately. If this is indeed the case, hydrogen solubility and the dissolved concentration of hydrogen are important. In our tests the amount of dissolved hydrogen was not monitored. Because the static pressure in the thruster conirols the solubility of hydrogen in is important to note that the static pressure was at all times $21.5 \pm 0.5$ psia.

\section{CONCLUSIONS}

1. A one-of-a-kind magnetohydrodynamic lest facility has been designed, fabricated, tested, and operated to provide unique data on seawater propulsion.

2. The test loop was modeled with both one and two dimensional models. The results of the tests indicate that the phenomena affecting the performance of marine MHD thrusters are well understood and can be adequately 
1.

quantified with relatively straight-forward analytical tools. Good agreement between measured and predicted data

- served to validate the developed NNS/ANL thiuster performance code.

3. The production of hydrogen at the cathode does not appear to have an impact on performance for the current densities relevant to full size thrusters.

4. The performance of the thruster is independent of the orientation of the magnetic field relative to gravity.

5. Bubble formation at the cathode (and presumably related noise) is dependent on both current density and fluid velocity.

\section{ACKNOWLEDGMENT}

This work has been supported by the U.S. Department of Energy under Contract W-31-1(19-Eng-38 through Argonne National Laboratory's Pilot Center for Superconductivity and Newport News Shipbuilding and Dry Dock Company.

The opinions expressed herein are those of the authors and do not necessarily reflect those of Argonne National Laboratory or Newport News Shipbuilding and Dry Dock Company.

\section{REFERENCES}

1. Phillips, O.M., "The Prospects for Magnetohydrodynamic Ship Propulsion". J. Ship Research, Vol. 43, March 1962, pp 43-51.

2. Doragh, R.A., "Magnetohydrodynamic Ship Propulsion Using Superconducting Magnets", Proc. Naval Arch. and Marine Engineers Transaction, Vol. 71, 1963.

3. Way, S., "Electromagnetic Propulsion for Cargo Submarines", Journal of Hydraulics, Vol. 2, No. 2, April 1.968, pp 49-57.

4. Saji, Y., Kitano, M., and Iwata, A., "Basic Study of Superconducting Electromagnetic Thrust Device for Propulsion in Seawater", Advances in Cryogenic Engineering (Timmerhaus, K.D. editor), Vol. 23, 1978, pp. 159-169.

5. Hummert, G.T., "An Evaluation of Direct Current Electromagnetic Propulsion In Seawater", Report ONR. CR 168-007-1, Westinghouse Research Laboratories, Pittsburgh, Pennsylvania, July 1979.

6. Doss, E.D., and Geyer, H.K., "The Need for Superconducting Magnets for MHD Seawater Propulsion", Proceedings of the $25^{\text {th }}$ Intersociety Energy Conversion Engineering Conference, August 1990, Vol. 5 pp. 540-545.

7. Ranellone, R.F., "Ship Integration and Construction Considerations", Proceedings of the International Symposium on Superconducting Magnetohydrodynamic Ship Propulsion, pp. 261-268.
8. Tempelmeyer, Kenneth E., "Electrical Characteristics of a Seawater MHD Thruster", DTRC Publication DTRC. 90/017, June 1990.

9. Doss, E.D. and Geyer, H.K., "An Overview of MHD Scawater Thruster Performance and Loss Mechanisms ${ }^{n}, 27^{\text {th }}$ Intersociety Energy Conversion Engineering Conference, August 1992. 


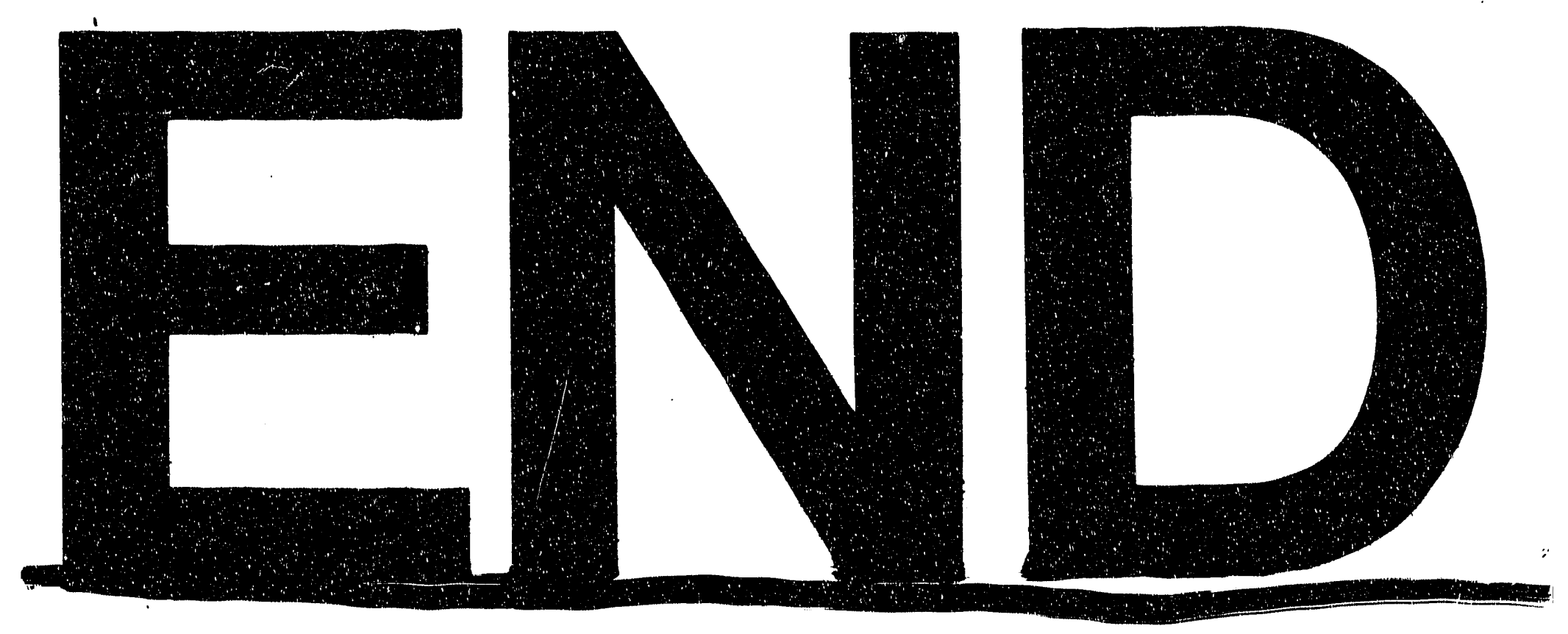

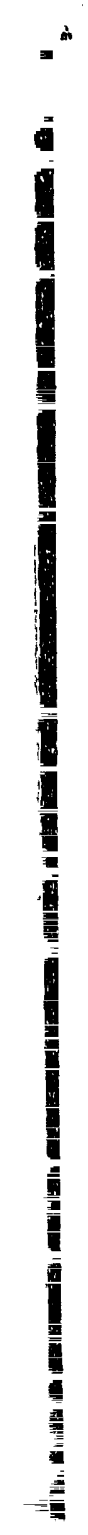
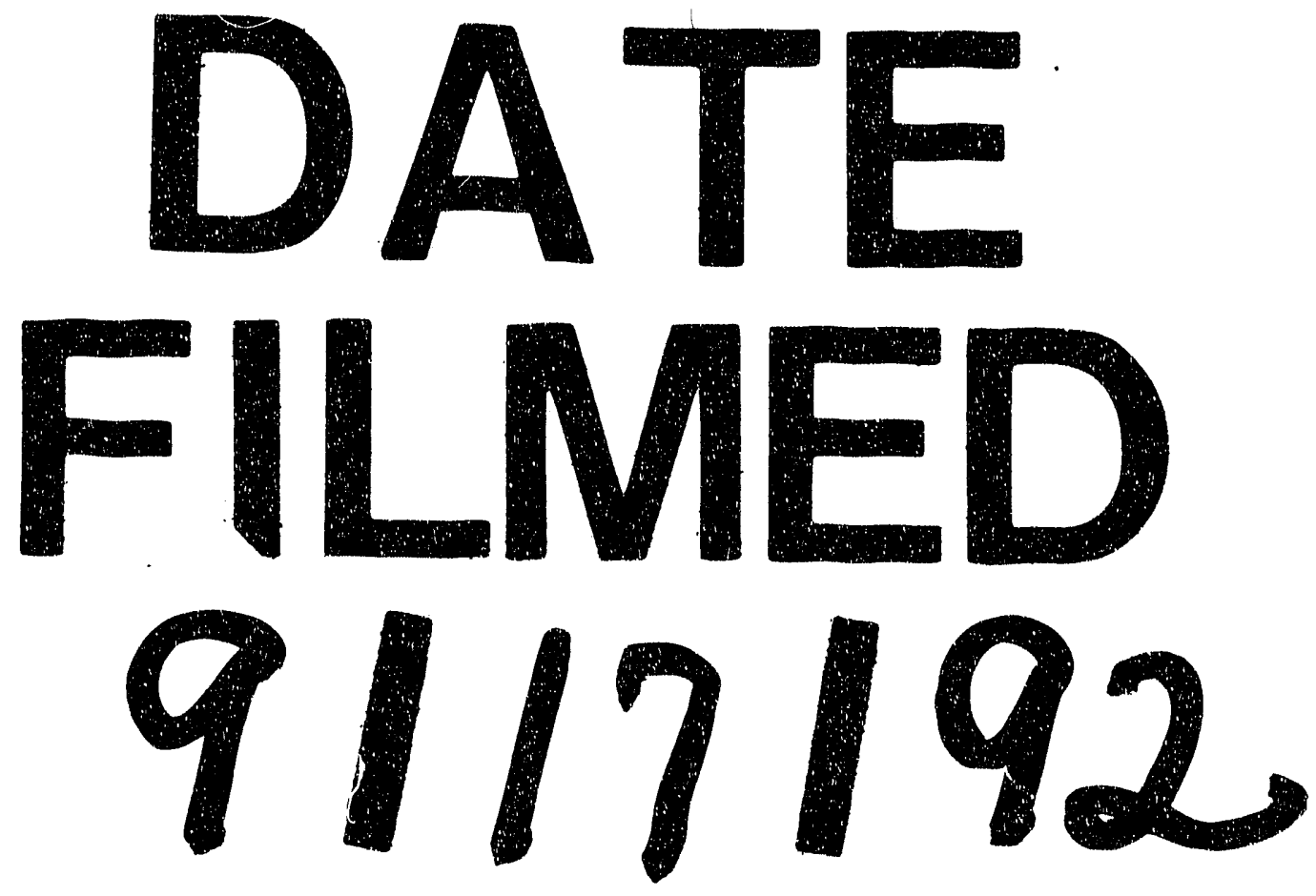
